\title{
Incentive Mechanisms to Prevent Efficiency Loss of Non-Profit Utilities
}

\author{
Carlos Barreto $^{\mathrm{a}, *}$, Eduardo Mojica-Nava ${ }^{\mathrm{b}}$, Nicanor Quijano ${ }^{\mathrm{c}}$ \\ ${ }^{a}$ Department of Electrical Engineering and Computer Science, Vanderbilt University, Nashville, TN, United States \\ ${ }^{b}$ Departamento de Ingeniería Eléctrica y Electrónica, Universidad Nacional de Colombia, Bogotá, Colombia \\ ${ }^{c}$ Departamento de Ingeniería Eléctrica y Electrónica, Universidad de los Andes, Bogotá, Colombia
}

\begin{abstract}
The modernization of the power system introduces technologies that may improve the system's efficiency by enhancing the capabilities of users. Despite their potential benefits, such technologies can have a negative impact. This subject has widely analyzed, mostly considering for-profit electric utilities. However, the literature has a gap regarding the impact of new technologies on non-profit utilities.

In this work, we quantify the price of anarchy of non-profit utilities, that is, the cost caused by lack of coordination of users. We find that users, in the worst case, can consume up to twice the optimal demand, obtaining a small fraction of the optimal surplus. For this reason, we leverage the theory of mechanism design to design an incentive scheme that reduces the inefficiencies of the system, which preserves the privacy of users. We illustrate with simulations the efficiency loss of the system and show two instances of incentive mechanism that satisfy either budget balance and budget deficit.
\end{abstract}

Keywords: Smart grid, demand response, electricity market, dynamic pricing, game theory.

\section{Introduction}

Power systems are experiencing a innovation process to improve multiple aspects, such as efficiency, reliability, and security. Although new technologies play an important role, the current modernization endeavor requires the active participation of customers. In particular, demand response programs leverage communication and automation technologies so that customers can manage their loads according to the system's state. In this way, customers can make better use of the available resources and offer services, such as help reducing the stress on the system or sell energy.

The literature on demand response focus mainly on how to achieve different goals, such as reduce demand peaks and/or improve the efficiency of the system 1, 2]. These research works often consider investor owned utilities, that is, firms focused on

\footnotetext{
* Corresponding author

Email addresses: carlos.a.barreto@vanderbilt.edu (Carlos Barreto), eamojican@unal.edu.co (Eduardo Mojica-Nava), nquijano@uniandes.edu.co (Nicanor Quijano)
}

maximizing their profits; however, little attention has been given to non-profit electric utilities (e.g., regulated or customer owned cooperatives).

Although investor owned utilities dominate the electricity markets, non-profit electric cooperatives play an important role in the economic development [3]. Besides, electric cooperatives have properties that foster the social support for renewable energy projects $4,5,6]$, 1 On one hand, cooperatives offer transparency in the prices and allow customers to control the source of energy. Thus, the owners/members of an electric cooperative can choose to support environmental or social objectives, rather than maximize profits, as investor owned firms.

On the other hand, cooperatives present the renewable energy infrastructures as community owned, which promotes their acceptance. Also, cooperatives can inform about environmental issues and give advice on how to reduce the environmental impact, e.g., reducing the consumption of energy 2

\footnotetext{
${ }^{1}$ The REScoop.eu network has 1500 renewable energy cooperatives in Europe [7].

${ }^{2}$ Other electric corporations profit with sales, so they do
} 
According to [6], the customers of Ecopower, a renewable energy cooperative from Belgium, reduced their average demand from $4000 \mathrm{kWh} /$ year to 3000 $\mathrm{kWh} /$ year within 3 years.

In this work we close a gap in the literature designing a demand response approach for non-profit utilities 3 First, we show that behavioral changes enabled with new technologies can harm the system's efficiency to a large degree. This motivates the design of an incentive scheme that reduces efficiency losses. This research is important for electric utilities, which face the challenge of adapting their policies to account for new capabilities of their customers.

\subsection{Literature Review}

The demand response literature often considers the following objectives: reduce or shift the demand peak, maximize the social welfare (the social satisfaction with an allocation of resources), minimize costs, or some combination of these goals [1, 8]. Maximizing the social welfare may encompass the other objectives, because 1) maximizing the social welfare, assuming inelastic demand, equals to minimizing costs, and 2) reduction of demand peaks may be necessary to improve the social welfare. However, these goals do not represent the objective of regulated or non-profit utilities, because they prioritize the customer's satisfaction over profits.

In general, both non-profit and regulated utilities may choose electricity tariffs so that their revenues amounts to the costs incurred to provide the service. The cost aggregates both the operating expenses (e.g., fuel, labor, and maintenance) and the cost of the capital invested (e.g., interest, debt, and a fair rate return to investors) 9]. In particular, the average cost pricing scheme guarantees that the utility collects the estimated total cost.

Game theory has been widely used in demand response studies to analyze interactions of selfish agents with conflicting interests $[10,11,12,13,14$, 15]. In this case, the conflict arises because customers may compete for a limited resource. Some research on demand response focus on setting up the conditions (e.g., prices, incentives) so that selfish agents reach a desired social goal. In other words, demand response attempts to coordinate

\footnotetext{
not have incentives to promote reductions in demand.

${ }^{3}$ Here we refer to non-profit utilities as customer owned utilities, regulate utilities, or electric cooperatives.
}

agents whose individual actions could harm the society.

The seminal work in [16] introduces the price of anarchy, a measure to estimate the impact of lack of coordination of agents. In our context, the price of anarchy can quantify the effect of new technologies that, although enhance the decision making capabilities of customers, may not improve their cooperation. Thus, we can think of demand response programs as efforts to reduce the price of anarchy (i.e., to improve the efficiency of the system).

Previous works analyze possible problems created by new technologies, such as stability 17, 18] or security issues [19, 20, 21, 22, 23]. In a way, these works analyze the cost of ill designed demand response schemes. However, the price of anarchy offers a different perspective, which measure the cost of not having demand response at all.

Some efforts have been made to analyzed the price of anarchy of different systems. For example, 24] analyzes the degradation of a network's performance due to unregulated traffic. Moreover, 25] studies how users who anticipate the effect of their actions reduce their utility in congestion games. However, we are not aware of works that analyze the price of anarchy of power systems with non-profit utilities.

The literature has several mechanisms to prevent efficiency losses in problems of resource allocation. For example, 26] proposes a resource allocation mechanism that has a bounded efficiency loss when users anticipate prices. On the other hand, 27] presents a pricing mechanism to implement efficient outcomes in smart grids. Although relevant, these works focus on maximizing the social welfare, that is, they consider for-profit firms. Moreover, 28] proposes the evolutionary implementation of a mechanism that guarantees efficiency and stability. This mechanism assumes that the payoff functions are additively separable in the private information of users.

Works such as 29, 30] propose decentralized mechanisms to allocate efficiently bandwidth in networks. These mechanisms satisfy the budget balance property 4 but require a multidimensional message space, because users must report their desired allocation and the price they are willing to pay for each link of the network.

\footnotetext{
${ }^{4}$ The mechanism does not need external subsidies nor imposes taxes on the users.
} 
Previous works analyze the dependence of the message space with the efficiency of the mechanism. For instance, 31] analyzes the minimum space required to implement optimal allocations in exchange economies, which is larger than the number of participants and resources. Furthermore, [32] shows that one-dimensional message spaces are unsuitable to achieve optimal allocations on contractive games (games whose best response is a contraction mapping).

\subsection{Contributions}

At a high level, we make the following contributions:

- We analyze the efficiency loss (price of anarchy) in power systems with non-profit utilities (most of the literature in demand response considers for profit-utilities).

- We propose an incentive mechanism to prevent inefficiencies and preserves the privacy of the customers.

- We illustrate the efficiency loss and the efficacy of the mechanism through simulations.

We leverage the theory of mechanism design to propose an incentive scheme that reduces the efficiency loss. Our incentives scheme is based on the Clarke pivot mechanism [33] and preserves private information using a one-dimensional message space per each resource to be allocated 5 We manage to design a mechanism that uses a one-dimensional space (to allocate a single resource) thanks to the properties of the system. This is possible because the wealth of each agent depends on their actions and the aggregate actions of other agents. Thus, we can decentralize decisions by sending to each agent the aggregated demand, which is one-dimensional. Likewise, users report their demand (a one dimensional signal) to the utility. The structure of the proposed mechanism is similar in philosophy to the mechanism in [34]; however, in our case users do not communicate their marginal payoff function.

We extend the works previously published in [35, 36] in several ways: 1) we find that the efficiency loss takes place with an overuse of resources

\footnotetext{
${ }^{5} \mathrm{~A}$ one-dimensional message space reduces the technical requirements of the system and also protects the privacy of the customers, since they do not report their electricity consumption preferences.
}

(in the inefficient equilibrium the demand can double the optimal value); 2) we prove that, in the worst case, the efficiency loss with strategic agents is arbitrarily large; 3) we show two instances of incentives that satisfy individual rationality (i.e., users have always positive surplus) and have either budget deficit or budget surplus; and 4) we generalize previous results relaxing restrictions on the price function, we only assume that the generation cost function is strictly convex.

\subsection{Organization of the Paper}

In Section 2 we introduce the electricity system model. We show that the average pricing scheme leads to optimal outcomes when users have limited capabilities, i.e., when they lack technologies to plan strategically their actions. Then, we show that strategic behaviors harm the systems efficiency. In particular, the main result of the paper shows that users have incentives to consume up to twice the optimal demand, which in turn reduces significantly the system's efficiency. In Section 3 we propose and analyze an incentives scheme to reach the optimal equilibrium with strategic users. We show two instances of the incentives that satisfy different properties. Finally, we conclude the paper in Section 4

\section{Background}

In this section we introduce a model of the power system 6 and analyze the system's equilibrium with two types of users, namely non-strategic and strategic, which describe users before and after the introduction of DR technologies, respectively. The main results of this section show that strategic behaviors damage the efficiency of the electricity system. In the worst case, strategic customers consume twice the optimal amount of energy, causing an arbitrarily large efficiency degradation.

\subsection{Notation}

We denote vectors with bold font. Let $\mathbf{q}$ be a vector of size $N$. Thus, we define $\boldsymbol{q}_{-i}$ as a vector with the elements of $\boldsymbol{q}$, except the $i^{t h}$, i.e., $\boldsymbol{q}_{-i}=$ $\left[q_{1}, \ldots, q_{i-1}, q_{i+1}, \ldots, q_{N}\right]$. The norm $\|\cdot\|$ represents

\footnotetext{
${ }^{6}$ Here we assume that a non-profit electric utility provides both generation, transmission, and distribution. Thus, the electric utility manages the whole system.
} 
Table 1: List of symbols.

\begin{tabular}{cl}
\hline Symbol & Description \\
\hline$N$ & Number of customers \\
$\mathcal{P}$ & Set of customers \\
$q_{i}$ & Demand of the $i^{\text {th }}$ customer \\
$\boldsymbol{q}$ & Demand of the population \\
$g$ & Total demand \\
$C$ & Generation cost function \\
$p$ & Unitary price of energy \\
$t_{i}$ & Payment of the $i^{t h}$ customer \\
$v_{i}$ & Valuation function \\
$U_{i}$ & Profit of the $i^{\text {th }}$ customer \\
$\sigma_{i}$ & Set of available strategies \\
$\mathcal{G}$ & Game \\
$\boldsymbol{\mu}$ & Optimal demand \\
$\boldsymbol{\xi}$ & Nash equilibrium \\
$r$ & Efficiency ratio \\
$\mathcal{M}$ & Mechanism \\
$I$ & Incentive function \\
$I^{d}$ & Incentive with budget deficit \\
$I^{s}$ & Incentive with budget surplus \\
$W_{i}$ & Profit with the incentives \\
$\mathcal{G}_{\mathcal{I}}$ & Game with incentives \\
$h$ & Estimation of the electricity price \\
$o$ & Outcome function \\
\hline &
\end{tabular}

the $L^{1}$-norm, that is, $\|\mathbf{q}\|=\sum_{i=1}^{N}\left|q_{i}\right|$. Finally, $\dot{f}$ and $\ddot{f}$ denote the first and second derivatives of the function $f$, respectively. Table 1 summarizes the symbols used in the document.

\subsection{Model of the Electricity System}

We consider a non-profit utility that provides energy to a population $\mathcal{P}=\{1, \ldots, N\}$ with $N$ customers. Let us denote by $C(g)$ the total cost to supply a demand of $g$ units of energy 7 For simplicity, we ignore transmission losses, therefore, the total energy generated equals the total demand, i.e., $g=\sum_{i \in \mathcal{P}} q_{i}$, where $q_{i} \geq 0$ is the demand of the $i^{\text {th }}$ user.

We define the profit of users as the benefit earned minus the cost of the energy consumed. Here we quantify the benefit of the $i^{\text {th }}$ user through a valuation function $v_{i}: \mathbb{R}_{>0} \rightarrow \mathbb{R}$, where $v_{i}\left(q_{i}\right)$ represents the monetary value that the user assigns to $q_{i}$ units

\footnotetext{
${ }^{7} \mathrm{C}(\mathrm{g})$ includes operation costs, such as generation, transmission, and a fair return for the utility.
}

of energy 8 Besides, the $i^{\text {th }}$ user pays $q_{i} p(g)$ for its consumption, where $p(g)$ is the unitary price of energy, which depends on the total demand $g$. In summary, the profit of the $i^{\text {th }}$ consumer is

$$
U_{i}\left(q_{i}, g\right)=v_{i}\left(q_{i}\right)-q_{i} p(g)
$$

We make the following assumption regarding the functions of the model.

\section{Assumption 1.}

1. The valuation function $v_{i}: \mathbb{R}_{\geq 0} \rightarrow \mathbb{R}$ is twice differentiable, concave, non-decreasing, and satisfies $v_{i}(0)=0$.

2. The cost function $C: \mathbb{R}_{\geq 0} \rightarrow \mathbb{R}_{\geq 0}$ is differentiable, strictly convex, and satisfies $C(g)>0$ if $g>0$.

3. The average cost $p(g)=\frac{C(g)}{g}$ is monotonically increasing, therefore, $\dot{p}(g) \geq 0$ for $g>0$.

The electricity tariff $p(\cdot)$ and the behavior of users determine the equilibrium of the system 9 In traditional power systems users cannot observe changes in prices, therefore, we assume that they cannot manifest strategic behaviors. However, new technologies grant users more information and capabilities to make decisions. Hence, users can develop strategies to anticipate the effect of their actions in the prices 33]. Below we show that the average cost price is the optimal tariff in systems with non-strategic users. Latter we prove that the selfinterest of strategic users can degrade the efficiency of the electricity system .

\subsection{System with Non-strategic Users}

Here we define the behavior of users before introducing DR technologies. Let us denote the surplus of non-strategic users as

$$
\tilde{U}_{i}\left(q_{i}, \tilde{p}\right)=v_{i}\left(q_{i}\right)-q_{i} \tilde{p}, \quad i \in \mathcal{P},
$$

where $\tilde{p} \in \mathbb{R}$ is the price observed by the users. Rational users will select the demand that maximizes

\footnotetext{
${ }^{8}$ The valuation function reflects the benefit consuming $q_{i}$ units of energy. For instance, a concave valuation function, such as $v_{i}\left(q_{i}\right)=\log \left(1+q_{i}\right)$, indicates that the value of an additional resource decreases with the total number of resources $q_{i}$.

${ }^{9}$ The equilibrium of a power system has the form of a Nash equilibrium, which describes situations where no user has incentives to change its demand unilaterally (see Definition 2).
} 
their surplus, given the constant price $\tilde{p}$ that they observe. Hence, each user solves the following optimization problem

$$
\begin{array}{cl}
\underset{q_{i}}{\operatorname{maximize}} & \tilde{U}_{i}\left(q_{i}, \tilde{p}\right)=v_{i}\left(q_{i}\right)-q_{i} \tilde{p} \\
\text { subject to } & q_{i} \geq 0 .
\end{array}
$$

From Assumption 1 the surplus in (11) is concave; hence, the previous problem has a unique solution, denoted by $\tilde{q}_{i}$, which satisfies [37, 38, 39]

$$
\begin{cases}\tilde{q}_{i}=0 & \text { if } \dot{v}_{i}(0)<\tilde{p}, \\ \dot{v}_{i}\left(\tilde{q}_{i}\right)-\tilde{p}=0 & \text { otherwise. }\end{cases}
$$

The previous expression implies that all users with positive demand $\left(\tilde{q}_{i}>0\right)$ have the same marginal valuation in the equilibrium.

The utility can select price schemes that allow users reach efficient demand profiles (or allocations of resources) acting independently. Below, we introduce the concept of Pareto optimal allocation and leverage the second welfare theorem to show that the average pricing scheme leads to a Pareto optimal allocation 10

\subsubsection{Pareto Optimality} [40]:

We define the Pareto efficiency criteria as follows

Definition 1. An allocation $\tilde{\boldsymbol{q}}=\left[\tilde{q}_{1}, \ldots, \tilde{q}_{N}\right]$ is Pareto optimal (or Pareto efficient), given some price $\tilde{p}$, if there is no other allocation $\hat{\boldsymbol{q}}$ such that $\tilde{U}_{i}\left(\hat{q}_{i}, \tilde{p}\right) \geq \tilde{U}_{i}\left(\tilde{q}_{i}, \tilde{p}\right)$ for all $i \in \mathcal{P}$, with strict inequality for some $i \in \mathcal{P}$.

From this definition, it follows that an allocation $\tilde{\boldsymbol{q}}$ that maximizes the consumer surplus, defined as $\sum_{i} \tilde{U}_{i}\left(\tilde{q}_{i}, \tilde{p}\right)$, is Pareto optimal. In other words, no other allocation $\hat{\boldsymbol{q}}$ would improve the benefit of users without affecting any of them, otherwise, $\hat{\boldsymbol{q}}$ would attain a higher social surplus than $\tilde{\boldsymbol{q}}$, which leads to a contradiction.

\subsubsection{Optimality of the Average Cost Pricing}

With the following optimization problem we express the problem of finding the system's equilibrium, represented by the tuple $(\tilde{p}, \tilde{\boldsymbol{q}})$, that maxi-

\footnotetext{
${ }^{10}$ In a Pareto optimal allocation no user can be made better off without another being made worse off.
}

mizes the customer surplus

$$
\begin{array}{cl}
\underset{q_{1}, \ldots, q_{N}, p}{\operatorname{maximize}} & \sum_{i \in \mathcal{P}} \tilde{U}_{i}\left(q_{i}, p\right) \\
\text { subject to } & C(\|\boldsymbol{q}\|)-\|\boldsymbol{q}\| p \leq 0, \\
& q_{i}\left(\dot{v}_{i}\left(q_{i}\right)-p\right)=0, \quad i \in \mathcal{P} \\
& q_{i} \geq 0, \quad i \in \mathcal{P} .
\end{array}
$$

The restriction in (3a) is a budget constraint required to guarantee that the total payments by the users $(\|\boldsymbol{q}\| p)$ cover the production costs $C(\|\boldsymbol{q}\|) 11$ Moreover, (3b) captures the optimal response of the users (conditions in (2)). The following result shows that a system with the average cost pricing has a Pareto efficient equilibrium that maximizes the customer surplus.

Theorem 1. Consider non-strategic users with surplus given by (11) and a generator with cost $C(\cdot)$ satisfying Assumption 1. Then, the average cost price function

$$
p(g)=C(g) / g,
$$

leads to a Pareto equilibrium.

The Appendix contains the proof of this and the remaining results.

\subsection{System with Strategic Users}

In this subsection we formulate the profit of strategic users as a function of both the total demand $g=\|\boldsymbol{q}\|$ and the user's demand $q$

$$
U_{i}\left(q_{i}, \boldsymbol{q}_{-i}\right)=v_{i}\left(q_{i}\right)-t_{i}(\boldsymbol{q}), \quad i \in \mathcal{P},
$$

where

$$
t_{i}(\boldsymbol{q})=q_{i} p(\|\boldsymbol{q}\|)
$$

represents the payment function of the $i^{\text {th }}$ user. We make the following assumption to guarantee that the profit of the individuals $U_{i}\left(q_{i}, \boldsymbol{q}_{-i}\right)$ is concave.

Assumption 2. The payment $t_{i}(\boldsymbol{q})$ in (6) is increasing convex with respect to $q_{i}$. Furthermore, the marginal payment $\frac{\partial t_{i}(\boldsymbol{q})}{\partial q_{i}}=p(\|\boldsymbol{q}\|)+q_{i} \dot{p}(\|\boldsymbol{q}\|)$ is increasing with respect to $q_{i}$.

\footnotetext{
${ }^{11}$ In practice, the operational restrictions of some generators (e.g., large start up costs) may force them to run even when they incur in losses, provided that they compensate the losses in future periods. Hence, the restriction in (3a refers to the long run operation 41].

12 Users must reveal their consumption only to the utility company, which in turn computes and broadcast the aggregate demand.
} 
As users improve their decision capabilities, the system's equilibrium changes; however, the system's efficiency may decrease. Before analyzing the efficiency of the system, we need introduce both the optimal equilibrium and the equilibrium that users reach pursuing their own interests. Later we investigate the relation between these equilibria.

\subsubsection{Optimal Equilibrium}

The demand $\boldsymbol{\mu} \in \mathbb{R}_{\geq 0}^{N}$ that maximizes the customer surplus (our efficiency metric), given the average cost price, solves the following optimization problem

$$
\begin{array}{ll}
\underset{q_{1}, \ldots, q_{N}}{\operatorname{maximize}} & \sum_{i \in \mathcal{P}} U_{i}\left(q_{i}, \boldsymbol{q}_{-i}\right)=\sum_{i \in \mathcal{P}} v_{i}\left(q_{i}\right)-C(\|\boldsymbol{q}\|) \\
\text { subject to } & q_{i} \geq 0, \quad i \in \mathcal{P} .
\end{array}
$$

From Assumption 1 the consumer surplus is strictly concave, therefore, there is a unique optimal solution that satisfies the following conditions 37]

$$
\begin{cases}\mu_{i}=0 & \text { if } \dot{v}_{i}(0)<\dot{C}(\|\boldsymbol{\mu}\|), \\ \dot{v}_{i}\left(\mu_{i}\right)=\dot{C}(\|\boldsymbol{\mu}\|) & \text { otherwise. }\end{cases}
$$

\subsubsection{Nash Equilibrium}

We analyze the interactions of users using the Cournot competition model, in which users individually and simultaneously select the quantity that they want to consume [42, 40]. Particularly, we define the game as a 3 -tuple $\mathcal{G}=\left\langle\mathcal{P},\left(\Sigma_{i}\right)_{i \in \mathcal{P}},\left(U_{i}\right)_{i \in \mathcal{P}}\right\rangle$, where $\mathcal{P}$ is the set of players (or customers), $\Sigma_{i}=$ $\mathbb{R}_{>0}$ is the set of available strategies (consumption of electricity) and $U_{i}: \Sigma_{1} \times \cdots \times \Sigma_{N} \rightarrow \mathbb{R}$ is the surplus function of the $i^{t h}$ player.

The equilibrium concept used in game theory is the Nash equilibrium (NE) [43, 44], defined as follows.

Definition 2. The Nash equilibrium of a game $\mathcal{G}$, denoted by $\boldsymbol{\xi} \in \mathbb{R}_{>0}^{N}$, satisfies $U_{i}\left(\xi_{i}, \boldsymbol{\xi}_{-i}\right) \geq$ $U_{i}\left(q_{i}, \boldsymbol{\xi}_{-i}\right)$, for all $q_{i} \in \overline{\mathbb{R}}_{\geq 0}$, for every user $i \in \mathcal{P}$.

From this definition we know that rational agents will choose $\xi_{i}$ when the other users choose $\boldsymbol{\xi}_{-i}$. In other words, $\xi_{i}$ is the demand that solves the following optimization problem

$$
\begin{array}{ll}
\underset{q_{i}}{\operatorname{maximize}} & U_{i}\left(q_{i}, \boldsymbol{\xi}_{-\boldsymbol{i}}\right) \\
\text { subject to } & q_{i} \geq 0
\end{array}
$$

From Assumption 2 we know that the problem in (8) is concave, and therefore, it has only one solution (given some $\boldsymbol{\xi}_{-i}$ ) that satisfies the following conditions

$$
\begin{cases}\xi_{i}=0 & \text { if } \dot{v}_{i}(0)<p(\|\boldsymbol{\xi}\|), \\ \dot{v}_{i}\left(\xi_{i}\right)=p(\|\boldsymbol{\xi}\|)+\xi_{i} \dot{p}(\|\boldsymbol{\xi}\|) & \text { otherwise. }\end{cases}
$$

Moreover, we can use the results by Rosen [45, Theorem 2] to prove that the Nash equilibrium of $\mathcal{G}$, denoted by $\boldsymbol{\xi}$, is unique.

\subsubsection{Electricity System Inefficiency}

From the equilibrium conditions in (7) and (9) we know that $\boldsymbol{\mu} \neq \boldsymbol{\xi}$. In other words, the user's individual interests are not aligned with the collective interests. Particularly, the following result shows that users consume more resources in the NE.

Theorem 2. Suppose that Assumption 1 is satisfied. Let $\boldsymbol{\mu}$ and $\boldsymbol{\xi}$ be the optimal and the Nash equilibrium (see (7) and (9)), respectively. Then, all users consume more resources in the NE, that is, $\xi_{i} \geq \mu_{i}$, for all $i \in \mathcal{P}$.

We use the concepts of the tragedy of the commons and the price of anarchy to analyze the efficiency loss 13 of the electricity system. On one hand, the tragedy of the commons is a situation in which the self interest of users leads to overuse of resources, despite of the harm to the society [46]. This occurs because the negative effects are shared by the community. Pitifully, the benefit of the community decreases when all individuals overuse resources. We define the tragedy of the commons as follows.

Definition 3 (Tragedy of the commons). At the optimal outcome, every agent has incentives to consume more resources.

Observe that the game $\mathcal{G}$ suffers the tragedy of the commons, since: i) every user has incentives to consume more than the optimal value of energy $\left(\xi_{i} \geq \mu_{i}\right)$; and ii) the self interest leads to an undesired social outcome. In particular, the next result shows that users can consume twice the optimal demand in the NE (when the price function $p(\cdot)$ is linear).

\footnotetext{
${ }^{13}$ Here we use the customer surplus as the efficiency metric; hence, the efficiency loss refers to the degradation of the customer surplus in the NE with respect to the optimal outcome.
} 
Lemma 1. Suppose that Assumption 1 is satisfied and assume that $\dot{C}(\cdot)$ is unbounded. If the users have the same valuation function with $\dot{v}_{i}(0)>p(0)$ and if the price function $p(\cdot)$ is linear, then the demand ratio $\|\boldsymbol{\xi}\| /\|\boldsymbol{\mu}\| \rightarrow 2$ as $N \rightarrow \infty$.

On the other hand, the price of anarchy is the ratio between the worst possible NE and the social optimum [47, 33. The next Theorem shows that the price of anarchy equals zero, that is, the customer surplus in the worst case NE is arbitrarily smaller than customer surplus in the social optimum. In contrast, systems with marginal cost prices, reduce their efficiency in at most $1 / 4$ [25].

Theorem 3. Suppose that Assumption 1 is satisfied and assume that $\dot{C}(\cdot)$ is unbounded . Let $\boldsymbol{\mu}$ and $\boldsymbol{\xi}$ be the optimal and the Nash equilibrium (see (7) and (9)), respectively. Then, the efficiency loss, that is, the ratio of the customer surplus with the worst case NE and that of the optimal outcome, satisfies

$$
r(\boldsymbol{\xi}, \boldsymbol{\mu})=\frac{\sum_{i} v_{i}\left(\xi_{i}\right)-\|\boldsymbol{\xi}\| p(\|\boldsymbol{\xi}\|)}{\sum_{i} v_{i}\left(\mu_{i}\right)-\|\boldsymbol{\mu}\| p(\|\boldsymbol{\mu}\|)} \geq 0,
$$

with equality when all users have the same linear valuation with $\dot{v}_{i}(0)>p(0), p(\cdot)$ is linear, and $N \rightarrow$ $\infty$.

Remark 1. According to Theorem 3, the worst case of efficiency loss occurs when $N \rightarrow \infty$. Here we are not suggesting that the population grows to infinity, but that the efficiency loss aggravates with the size of the population.

We can model the impact of inelastic loads by setting a minimum demand for each user (to account for the demand of appliances that do not react to prices). The total demand with these restrictions, denoted $\|\hat{\boldsymbol{\mu}}\|$, may be higher than the optimal demand without restrictions, that is, $\|\boldsymbol{\mu}\| \leq\|\hat{\boldsymbol{\mu}}\| \leq$ $\|\boldsymbol{\xi}\|$. Thus, we can conjecture that inelastic loads can reduce the efficiency loss, because they can reduce the distance between the optimal and the inefficient demand.

Example 1. Let us illustrate loss of efficiency of the customer surplus as a function of the number of users $N$. Here we use the following linear valuation function

$$
v_{i}\left(q_{i}\right)=\alpha_{i} q_{i},
$$

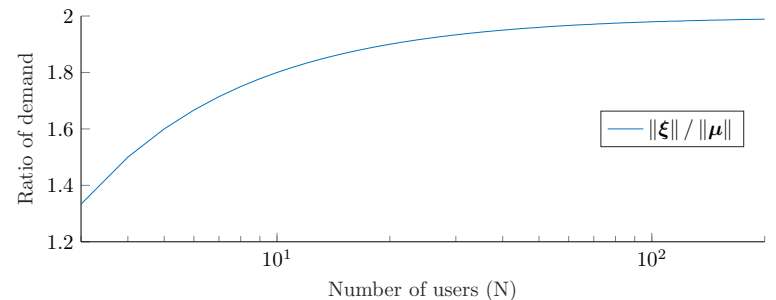

(a) Ratio of demand $\|\boldsymbol{\xi}\| /\|\boldsymbol{\mu}\|$.

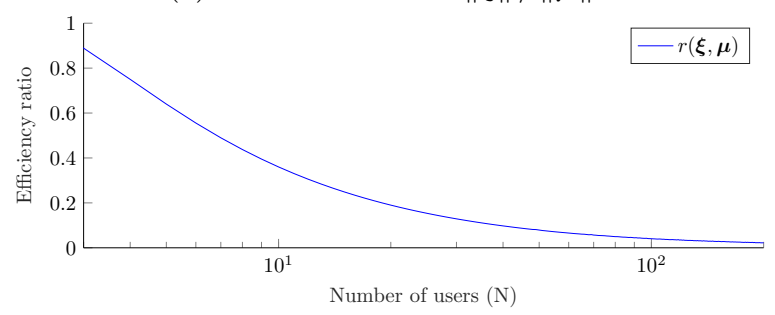

(b) Efficiency ratio $r(\boldsymbol{\xi}, \boldsymbol{\mu})$.

Figure 1: Ratio of demand and efficiency ratio as a function of the number of users $N$. In the worst case the demand ratio tends to 2 and the efficiency ratio (see (10) tends to 0 as $N \rightarrow \infty$.

where the parameter $\alpha_{i}$ characterizes the valuation of the $i^{\text {th }}$ agent (we select $\alpha_{i}=10$ for all $i \in \mathcal{P}$ ). On the other hand, we define a quadratic generation cost function $C(q)=\beta q^{2}+b q$, and the unitary price function as $p(g)=C(g) / g=\beta g+b$, where $\beta=1$ and $b=1$. Since all users have the same valuation function and $\dot{v}_{i}(0)<p(0)$, then the individual demand is positive (see (9)).

Fig. 1 shows the ratio of demand and efficiency ratio as a function of the number of users $N 14$ On one hand, Fig. 1a shows that the demand ratio $\|\boldsymbol{\xi}\| /\|\boldsymbol{\mu}\|$ closes to 2 for large populations, as expected from Lemma 1. On the other hand, Fig. 1b shows that the efficiency ratio $r(\boldsymbol{\xi}, \boldsymbol{\mu})$ tends to zero, confirming the worst efficiency loss from Theorem 3 .

These results show that lack of coordination harms the efficiency of the system. In particular, the users can consume up to twice the optimal demand and obtain a small fraction of the optimal surplus. Next we propose an incentive scheme that mitigates the system's inefficiencies.

\footnotetext{
${ }^{14}$ Section 5.5 has details on the optimization method used in the experiments.
} 


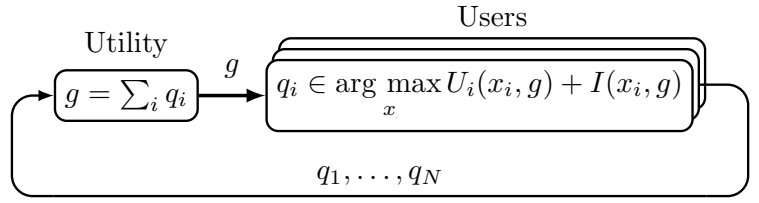

Figure 2: Scheme of a decentralized price mechanism. The utility broadcasts the total demand so that each agent can determine its optimal demand.

\section{Incentives-Based Mechanism}

The efficiency loss of the Nash equilibrium motivates the design of an incentives mechanism to improve the equilibrium in strategic environments [40, 48]. In this section, we introduce a price mechanism that improves the efficiency of the system when users are strategic. Also, we show two instances of the mechanism with different properties.

From the perspective of mechanism design, the principal (in this case the utility) can design the payoff structure of the game to reach a desired social goal. Hence, the principal intervenes to guarantee that selfish users reach an equilibrium that maximizes the customer surplus; this considering the private information held by each agent, necessary to calculate the optimal outcome. Summarizing, mechanism design consists in designing a solution system to a decentralized optimization problem with private information [48]. A mechanism $\mathcal{M}=\left\{\Sigma_{1}, \ldots, \Sigma_{N}, o(\cdot)\right\}$ defines a set of strategies $\Sigma_{i}$ for each player and an outcome rule $o: \Sigma_{1} \times \ldots \times \Sigma_{N} \rightarrow \mathcal{O}$ that maps from the set of possible strategies to the set of possible outcomes $\mathcal{O}$ [40].

Here we propose an indirect revelation mechanism, which avoids the revelation of private information, and uses a one-dimensional message space (see Fig. 2). In this mechanism, the users communicate their demand $q_{i}$ to the principal, who in turn calculates and broadcasts the total demand $\|\boldsymbol{q}\|$ to the users 15 Then, users use the incoming information to adjust their demand. In this mechanism the users have the same strategy space $\left(\left(\Sigma_{i}\right)_{i \in \mathcal{P}}\right)$ as in the original system (game $\mathcal{G}$ ).

\footnotetext{
15 We assume that the utility's infrastructure prevent users from tampering their electricity meters to misreport their demand. Nonetheless, the principal can calculate the total demand indirectly, inquiring the total generated energy. In other words, the principal can use the feedback of the physical world to gather information.
}

The outcome rule $o(\cdot)$ of the mechanism returns the payment for each user; hence, $o(\cdot)=$ $\left[o_{1}(\cdot), \ldots, o_{N}(\cdot)\right]$ and the outcome space is $\mathcal{O} \equiv \mathbb{R}_{>0}^{N}$. The outcome rule $o(\cdot)$ must guarantee that the game has a NE equal to the optimal outcome $\boldsymbol{\mu}$. To this end, we modify the payment function $t_{i}(\cdot)$ adding an incentive $I_{i}(\cdot)$ that aligns the users' profit with the population's objective function (customer surplus). We achieve this by selecting a function $I_{i}(\cdot)$ that estimates the externalities (or the impact on prices) caused by the $i^{t h}$ user.

Let the payment of the $i^{\text {th }}$ user be

$$
o_{i}(\boldsymbol{q})=t_{i}(\boldsymbol{q})+I_{i}(\boldsymbol{q}),
$$

with incentives of the form

$$
I_{i}(\boldsymbol{q})=I\left(q_{i},\|\boldsymbol{q}\|\right)=\left\|\boldsymbol{q}_{-i}\right\|\left(h\left(\left\|\boldsymbol{q}_{-i}\right\|\right)-p(\|\boldsymbol{q}\|)\right),
$$

where $\left\|\boldsymbol{q}_{-i}\right\|=\|\boldsymbol{q}\|-q_{i}$ and the function $h\left(\left\|\boldsymbol{q}_{-i}\right\|\right)$ estimates the electricity price without the $i^{t h}$ user in the system. We choose this incentive structure because each user only knows its own demand $q_{i}$ and the total demand $\|\boldsymbol{q}\|$, ignoring the precise demand of other users. Furthermore, we assume that users are indistinguishable in the system; hence, two users with the same demand impose the same externalities to the system. The form of this incentive is related to the price used in the VCG mechanism [33] and some payoff functions used in potential games [49].

Introducing the incentives in (12) we obtain a new game defined as the 3-tuple $\mathcal{G}_{\mathcal{I}}=$ $\left\langle\mathcal{P},\left(\Sigma_{i}\right)_{i \in \mathcal{P}},\left(W_{i}\right)_{i \in \mathcal{P}}\right\rangle$, where $\mathcal{P}$ is the set of players, $\Sigma_{i}$ is the set of available strategies of each player, and $W_{i}: \Sigma_{1} \times \cdots \times \Sigma_{N} \rightarrow \mathbb{R}$ is the surplus function of the $i^{\text {th }}$ player, defined as

$$
\begin{gathered}
W_{i}\left(q_{i}, \boldsymbol{q}_{-i}\right)=v_{i}\left(q_{i}\right)+o_{i}(\boldsymbol{q})=U_{i}\left(q_{i}, \boldsymbol{q}_{-i}\right)+I_{i}(\boldsymbol{q}) \\
=v_{i}\left(q_{i}\right)-\|\boldsymbol{q}\| p(\|\boldsymbol{q}\|)+\left\|\boldsymbol{q}_{-i}\right\| h_{i}\left(\left\|\boldsymbol{q}_{-i}\right\|\right)
\end{gathered}
$$

Observe that the NE of the game $\mathcal{G}_{\mathcal{I}}$ has the same equilibrium conditions than the optimal outcome in Section 2.4.1 hence, the $\mathrm{NE}$ of $\mathcal{G}_{\mathcal{I}}$ is equal to the optimal equilibrium $\boldsymbol{\mu}$.

\subsection{Mechanism's Properties}

Now we analyze the properties of the incentive mechanism and provide of two instances that have different properties. First, let us define some desirable properties of mechanisms, namely budget balance and individual rationality. 


\subsubsection{Budget Balance}

It is desirable to make the net payments equal to zero, that is, guarantee that the sum of charges is equal to the total cost [33]. In this way the system doesn't require external subsidies nor imposes taxes on the population. This property is known as budget balance, and is defined as follows

Definition 4. A mechanism that implements payments $o_{i}(\cdot)$ is budget balanced if $\sum_{i \in \mathcal{P}} o_{i}(\boldsymbol{q})=$ $C(\|\boldsymbol{q}\|)$.

Note that the original game $(\mathcal{G})$ satisfies the budget balance property, because the total payments equal the generation cost (see (44)). Therefore, we need that $\sum_{i \in \mathcal{P}} I_{i}(\boldsymbol{q})=0$ to preserve the budget balance in $\mathcal{G}_{\mathcal{I}}$. However, the next theorem shows that it is impossible to find a function $h(\cdot)$ that balances the amount of rewards (price discounts) and penalties (price increment) imposed to customers. Consequently, the mechanism requires either inflow or outflow of resources.

Theorem 4. Suppose that Assumption 1 is satisfied and let $p(\cdot)$ be the average cost price function. Then there is no function $h(\cdot)$ such that a mechanism with payments defined by (11) satisfies the budget balance property.

This result is an analogous to the MyersonSatterthwaite impossibility theorem [50], which states the impossibility of designing a mechanisms with ex-post efficiency and without external subsidies in games between two parties. However, Theorem 4 considers a particular case with 1) nonlinear prices (subject to Assumption 11) and 2) the customer surplus as the maximization criteria, rather than the aggregate surplus.

\subsubsection{Individual Rationality}

The utility company cannot force users to sign contracts accepting the incentives scheme. Hence, the mechanism must ensure that every user voluntarily decides to join the system with incentives (game $\mathcal{G}_{\mathcal{I}}$ ). In particular, a rational user would accept a mechanism if it guarantees higher benefits participating. This property is called individual rationality and is defined as

Definition 5. A mechanism is individual rational if the surplus at the equilibrium is larger than zero, that is, $W_{i}(\boldsymbol{\mu}) \geq 0$.
The following result shows the conditions to guarantee that the proposed mechanism satisfies the individual rationality.

Proposition 1. Let Assumption 1 be satisfied. Consider a mechanisms with payments given by (11). If $h\left(\left\|\boldsymbol{\mu}_{-i}\right\|\right)-p\left(\left\|\boldsymbol{\mu}_{-i}\right\|\right) \geq 0$, then the mechanism is individually rational.

\subsection{Examples of Incentives}

Here we present two instances of individual rational incentives that satisfy either budget deficit or surplus. On one hand, the following result shows an instance of a mechanism that has budget deficit, that is, $\sum_{i \in \mathcal{P}} o_{i}(\boldsymbol{q}) \leq 0$. This implies that the mechanism requires external subsidies to operate.

Proposition 2. Let Assumption 1 be satisfied. A mechanism with payments given by (11) with

$$
h(g)=p(g)
$$

satisfies the individual rationality property and has budget deficit, i.e., $\sum_{i \in \mathcal{P}} o_{i}(\boldsymbol{q}) \leq C(\|\boldsymbol{q}\|)$ (or equivalently $\left.\sum_{i \in \mathcal{P}} I_{i}(\boldsymbol{q}) \leq 0\right)$.

On the other hand, the following result shows a mechanism that has budget surplus, that is, $\sum_{i \in \mathcal{P}} I_{i}(\boldsymbol{q}) \geq 0$, which implies that the mechanism imposes taxes on users.

Proposition 3. Let Assumption 1 be satisfied. A mechanism with payments given by (11) with

$$
h(g)=p\left(\frac{N}{N-1} g\right)
$$

satisfies the individual rationality property and has budget surplus, i.e., $\sum_{i \in \mathcal{P}} o_{i}(\boldsymbol{q}) \geq C(\|\boldsymbol{q}\|)$ (or equivalently $\sum_{i \in \mathcal{P}} I_{i}(\boldsymbol{q}) \geq 0$ ).

Table 2 shows a summary of the properties of incentives with $h(\cdot)$ given by (13) and (14), denoted $I^{d}(\cdot)$ and $I^{s}(\cdot)$, respectively.

Example 2. Let us illustrate the effect of incentives in the customer surplus as a function of the number of users. In this case we use nonlinear valuation functions of the form

$$
v_{i}\left(q_{i}\right)=\alpha_{i} \log \left(1+q_{i}\right),
$$

with

$$
\alpha_{i}=10+\frac{i-1}{N-1} .
$$


Table 2: Properties of the mechanisms.

\begin{tabular}{lll}
\hline & $h(g)=p\left(\frac{N}{N-1} g\right)$ & $h(g)=p(g)$ \\
\hline If $q_{i}=q_{j}, \forall i, j \in \mathcal{P}$ & $I_{i}^{s}(\boldsymbol{q})=0$ & $I_{i}^{d}(\boldsymbol{q}) \leq 0$ \\
Weak budget balance & $\sum_{i \in \mathcal{P}} I_{i}^{s}(\boldsymbol{q}) \geq 0$ & $\sum_{i \in \mathcal{P}} I_{i}^{d}(\boldsymbol{q}) \leq 0$ \\
Individual rationality & $W_{i}(\boldsymbol{\mu}) \geq 0$ & $W_{i}(\boldsymbol{\mu}) \geq 0$ \\
\hline
\end{tabular}

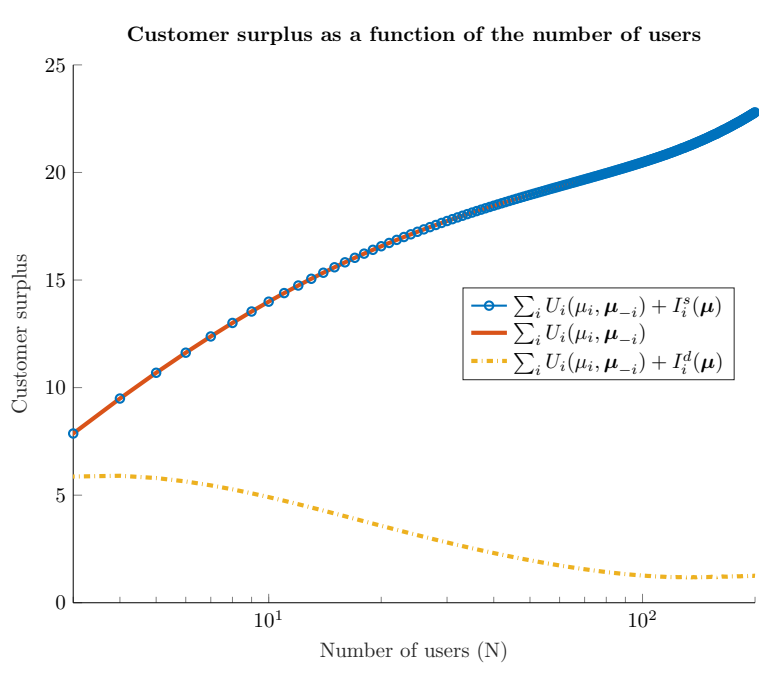

Figure 3: Customer surplus with and without incentives for different population sizes. The mechanism with budget deficit generates less profit for the users, but the profit is positive.

If the unitary price function is $p(g)=C(g) / g=$ $\beta g+b$, then the incentives from Table 0 are

$$
I_{i}^{s}(\boldsymbol{q})=\left\|\boldsymbol{q}_{-i}\right\| \frac{\beta}{N-1}\left(\left\|\boldsymbol{q}_{-i}\right\|-q_{i}(N-1)\right)
$$

and

$$
I_{i}^{d}(\boldsymbol{q})=-\left\|\boldsymbol{q}_{-i}\right\| \beta q_{i}
$$

Fig. 3 shows that the mechanism with budget deficit $\left(I^{d}\right)$ generates less profit for the society, but the profit is positive (because the mechanism is individual rational). Furthermore, the mechanism with budget surplus $\left(I^{s}\right)$ does not have a notorious effect in the customer surplus (in the simulations $\left.0 \leq \sum_{i \in \mathcal{P}} I_{i}^{s}(\boldsymbol{q}) \leq 0.0983\right)$.

\section{Conclusions and Future Directions}

In this work we find that, without a proper coordination, users who become strategic (thanks to new technologies) can harm the efficiency of the power system. Particularly, with the average cost prices, the efficiency loss can be arbitrarily large, while systems that use marginal cost prices have an efficiency loss of at most 1/4 [25]. Furthermore, in the worst case NE the users consume twice the optimal energy.

We propose an indirect revelation mechanism to improve the efficiency of the system. Our mechanism uses a one dimensional message space and avoids revelation of private information. We show two instances of the mechanism that satisfy either budget deficit or budget surplus. Furthermore, the properties of the mechanism guarantee that users would join the incentives program voluntarily, since they have non-negative surplus.

In this work, we use a stylized power system model to facilitate the analysis, however, it is important to consider the stochastic nature of both demand and generation and temporal interdependencies of the demand.

\section{Acknowledgements}

The paper has benefited substantially from conversations with Ramesh Johari and the comments from anonymous reviewers. This work has been supported in part by Project SGR C/marca 2015.

\section{Appendix}

In this section we include the proofs of the results introduced in the paper.

\subsection{Optimal Price Function in Non-strategic Set- tings}

The following proof demonstrates that the average cost price function maximizes the customer surplus when users are non-strategic.

Proof of Theorem 1. The proof has two steps. First, we show that the system's equilibrium $(\tilde{p}, \tilde{\boldsymbol{q}})$, 
with $\tilde{p}=C(\|\tilde{\boldsymbol{q}}\|) /\|\tilde{\boldsymbol{q}}\|$ (the average cost evaluated at the efficient outcome $\tilde{\boldsymbol{q}}$ ) maximizes the customer surplus of non-strategic users (see (3) ). However, the optimal price $\tilde{p}$, which is constant, depends on the optimal allocation, which is unknown beforehand. In the second step we show that a power system with the average cost price function $p(\|\boldsymbol{q}\|)=C(\|\boldsymbol{q}\|) /\|\boldsymbol{q}\|$ clears with the efficient allocation $\tilde{\boldsymbol{q}}$. In other words, with the average cost price function the system's equilibrium maximizes the customer surplus.

Step (i): Let us define the restrictions of the optimization problem in (3) as

$$
\Psi(\boldsymbol{q}, p)=C(\|\boldsymbol{q}\|)-\|\boldsymbol{q}\| p
$$

and

$$
\Phi_{i}\left(q_{i}, p\right)=q_{i}\left(\dot{v}_{i}\left(q_{i}\right)-p\right),
$$

where $(p, \boldsymbol{q})$ is a system's equilibrium. The Lagrangian of the problem in (3) is

$$
\begin{aligned}
\mathcal{L}(\boldsymbol{q}, p, \boldsymbol{\lambda}, \boldsymbol{\eta})=\sum_{i} \tilde{U}_{i}\left(q_{i}, p\right)-\lambda_{0} \Psi(\boldsymbol{q}, p) \\
-\sum_{i} \eta_{i} \Phi_{i}\left(q_{i}, p\right)+\sum_{i} \lambda_{i} q_{i}
\end{aligned}
$$

where $\boldsymbol{\lambda}=\left[\lambda_{0}, \ldots, \lambda_{N}\right]$ and $\boldsymbol{\eta}=\left[\eta_{1}, \ldots, \eta_{N}\right]$. The tuple that maximizes the customer surplus, denoted $(\tilde{p}, \tilde{\boldsymbol{q}})$, satisfies the following first order necessary conditions 38, 39]

$$
\frac{\partial \mathcal{L}(\tilde{\boldsymbol{q}}, \tilde{p}, \boldsymbol{\lambda}, \boldsymbol{\eta})}{\partial p}=\sum_{i} \tilde{q}_{i}\left(\lambda_{0}-1\right)+\sum_{i} \eta_{i} \tilde{q}_{i}=0,
$$

$\frac{\partial \mathcal{L}(\tilde{\boldsymbol{q}}, \tilde{p}, \boldsymbol{\lambda}, \boldsymbol{\eta})}{\partial q_{i}}=\phi_{i}-\lambda_{0} \psi-\eta_{i}\left(\phi_{i}+\tilde{q}_{i} \ddot{v}_{i}\left(\tilde{q}_{i}\right)\right)+\lambda_{i}=0$

$$
\begin{gathered}
\Phi_{i}\left(\tilde{q}_{i}, \tilde{p}\right)=0, \\
\lambda_{0} \Psi(\tilde{\boldsymbol{q}}, \tilde{p})=0,
\end{gathered}
$$

$$
\lambda_{i} \tilde{q}_{i}=0
$$

with $\phi_{i}=\dot{v}_{i}\left(\tilde{q}_{i}\right)-\tilde{p}, \psi=\dot{C}(\|\tilde{\boldsymbol{q}}\|)-\tilde{p}, \lambda_{k} \geq 0$, and $\eta_{i} \geq 0$, for $i \in\{1, \ldots, N\}$ and $k \in\{0, \ldots, N\}$.

Let us assume by contradiction that the cost restriction (15) does not bind. Therefore, $\Psi(\tilde{\boldsymbol{q}}, \tilde{p}) \leq 0$, which implies that $\tilde{p} \geq C(\|\tilde{\boldsymbol{q}}\|) /\|\tilde{\boldsymbol{q}}\|>0$ (the rightmost inequality follows because $C(\|\tilde{\boldsymbol{q}}\|)>0$ if $\|\tilde{\boldsymbol{q}}\|>0$ ). Thus, we need $\lambda_{0}=0$ to satisfy (19). Moreover, for every user $i$ with $\tilde{q}_{i}>0$ we have $\lambda_{i}=0$ and $\phi_{i}=0$ to satisfy (20) and $\Phi_{i}\left(\tilde{q}_{i}, \tilde{p}\right)=0$ (see the equality constraint in (16)). Also, when $\tilde{q}_{i}>0, \eta_{i}$ must be positive to satisfy (17).

With the previous considerations (18) becomes

$$
\eta_{i} \tilde{q}_{i} \ddot{v}_{i}\left(\tilde{q}_{i}\right)=0 .
$$

Observe that if the valuation function $v_{i}(\cdot)$ is concave, then $\ddot{v}_{i}\left(\tilde{q}_{i}\right) \leq 0$. Thus, (21) holds only if $\ddot{v}_{i}\left(\tilde{q}_{i}\right)=0$; however, since $\phi_{i}=0$, we know that $\dot{v}_{i}\left(\tilde{q}_{i}\right)=\tilde{p}=0$. This leads to a contradiction, because $\tilde{p} \geq C(\|\tilde{\boldsymbol{q}}\|) /\|\tilde{\boldsymbol{q}}\|>0$. In conclusion, the cost constraint in (15) must bind in the equilibrium that maximizes the customer surplus. Hence, the price at the equilibrium is equal to the average cost.

Step (ii): Now, let us use the average cost pricing function $p(\|\boldsymbol{q}\|)=C(\|\boldsymbol{q}\|) /\|\boldsymbol{q}\|$ instead of a constant price. Here we intend to prove that the system has only one equilibrium, which corresponds to the Pareto optimal allocation.

Let us assume by contradiction that the system has two equilibria, namely the optimal allocation $\tilde{\boldsymbol{q}}$ and a different allocation $\hat{\boldsymbol{q}}$ with $\tilde{q}_{i} \neq \hat{q}_{i}$ for some $i \in \mathcal{P}$. First, let us assume that $\tilde{q}_{i}>\hat{q}_{i}>0$, then, $\dot{v}_{i}\left(\tilde{q}_{i}\right)<\dot{v}_{i}\left(\hat{q}_{i}\right)$. Our previous hypothesis, together with the optimal response of users (see (2)) and the average cost pricing, leads to

$$
\dot{v}_{i}\left(\tilde{q}_{i}\right)=\tilde{p}(\|\tilde{\boldsymbol{q}}\|)<\dot{v}_{i}\left(\hat{q}_{i}\right)=\hat{p}(\|\hat{\boldsymbol{q}}\|) .
$$

Since all users have the same marginal valuation, the previous expression holds for every user with positive demand. This implies that $\tilde{q}_{i}>\hat{q}_{i}$ for every $i$ with $\hat{q}_{i}>0$; hence, we conclude that $\|\tilde{\boldsymbol{q}}\|>\|\hat{\boldsymbol{q}}\|$. Observe that (22) is true if the tariff $p(\cdot)$ strictly decreases with the total consumption, which contradicts Assumption 1 .

Hence, our initial assumption that $\tilde{q}_{i}>\hat{q}_{i}$ is false. Furthermore, we can construct a similar argument to show that $\tilde{q}_{i}<\hat{q}_{i}$ is false as well. Therefore, the system with the average pricing function allows only one equilibrium, which corresponds to the efficient allocation.

\subsection{Efficiency Loss in Strategic Settings}

This proof shows that the optimal equilibrium and the NE in strategic settings are distinct; hence, this result shows that the system's NE is inefficient. The next section investigates the efficiency loss in the NE with respect to the optimal equilibrium.

Proof of Theorem 2. In this proof we show first that $\|\boldsymbol{\mu}\| \leq\|\boldsymbol{\xi}\|$ and then we prove that $\mu_{j} \leq \xi_{j}$ for all $j$. 
First, let us assume by contradiction that $\|\boldsymbol{\xi}\|<$ $\|\boldsymbol{\mu}\|$, hence, there exist some $j$ such that $\mu_{j}>\xi_{j}>$ 0 with the following equilibrium condition (see (7))

$$
\dot{v}_{j}\left(\mu_{j}\right)=\dot{C}(\|\boldsymbol{\mu}\|) \geq \dot{C}(\|\boldsymbol{\xi}\|),
$$

where $\dot{C}(g)=p(g)+g \dot{p}(g)$ (see (4)). We can use the equilibrium conditions in (9) to express the previous inequality as

$$
\dot{v}_{j}\left(\mu_{j}\right) \geq \dot{v}_{j}\left(\xi_{j}\right)+\left(\|\boldsymbol{\xi}\|-\xi_{j}\right) \dot{p}(\|\boldsymbol{\xi}\|) .
$$

Since $\mu_{j}>\xi_{j}$, then $\dot{v}_{j}\left(\mu_{j}\right)<\dot{v}_{j}\left(\xi_{j}\right)$, because $v_{i}(\cdot)$ is concave and increasing; hence, (23) is true if $(\|\boldsymbol{\xi}\|-$ $\left.\xi_{j}\right) \dot{p}(\|\boldsymbol{\xi}\|)<0$, which is not possible, since $\|\boldsymbol{\xi}\|-$ $\xi_{i} \geq 0$ and $\dot{p}(\cdot)>0$ (see Assumption 1). From this contradiction we conclude that $\|\boldsymbol{\xi}\| \geq\|\boldsymbol{\mu}\|$.

Now, let us assume by contradiction that there exists some $j$ such that $\mu_{j}>\xi_{j}$. With the same argument used at the beginning of the proof we conclude that

$$
\begin{aligned}
\dot{v}_{j}\left(\mu_{j}\right)=\dot{C}(\|\boldsymbol{\mu}\|) & \leq \dot{C}(\|\boldsymbol{\xi}\|) \\
& =\dot{v}_{j}\left(\xi_{j}\right)+\left(\|\boldsymbol{\xi}\|-\xi_{j}\right) \dot{p}(\|\boldsymbol{\xi}\|) .
\end{aligned}
$$

However, the previous expression requires that $\left(\|\boldsymbol{\xi}\|-\xi_{j}\right) \dot{p}(\|\boldsymbol{\xi}\|)<0$, which is not possible. Therefore, we conclude that $\mu_{j} \leq \xi_{j}$ for all $j \in \mathcal{P}$.

\subsection{Price of Anarchy}

Here we undertake the task of investigating the price of anarchy when users become strategic. First, we analyze how the total demand changes as the number of users increases. Later, we find the conditions in which the ratio of demand $\|\boldsymbol{\xi}\| /\|\boldsymbol{\mu}\|$ reaches the upper bound. These results allow us to prove that the efficiency loss is arbitrarily large.

The first result shows that the total demand of the system cannot decrease as the number of users increases. In particular, it can occur that only a subset of users with high valuations can afford the electricity prices (see (9)). Hence, the demand does not necessarily increase as the population grows.

Proposition 4. Suppose that Assumption 1 is satisfied and let $\boldsymbol{\xi}$ and $\hat{\boldsymbol{\xi}}$ be the Nash equilibrium of two systems with $\mathcal{P}$ and $\hat{\mathcal{P}}$ users, respectively. If $\mathcal{P} \subseteq \hat{\mathcal{P}}$, then $\|\boldsymbol{\xi}\| \leq\|\hat{\boldsymbol{\xi}}\|$.

Proof. We assume by contradiction that the total demand in the NE strictly decreases with the number of users, that is, $\|\boldsymbol{\xi}\|>\|\hat{\boldsymbol{\xi}}\|$. Hence, at least one user $j \in \mathcal{P}$ reduces its demand, that is, there exists some $j$ such that $\xi_{j}>\hat{\xi}_{j}>0$. From (9) we know that $\hat{\xi}_{j}$ satisfies

$$
\dot{v}_{j}\left(\hat{\xi}_{j}\right)=\dot{t}_{j}(\hat{\boldsymbol{\xi}})=p(\|\hat{\boldsymbol{\xi}}\|)+\hat{\xi}_{j} \dot{p}(\|\hat{\boldsymbol{\xi}}\|) .
$$

The right hand side of (24) is increasing with respect to $\hat{\xi}_{j}$ (see Assumption 1). Therefore, we can find an upper bound of $\dot{v}_{j}\left(\hat{\xi}_{j}\right)$ replacing in the right hand side of (24) $\hat{\xi}_{j}$ by $k_{j}=\delta+\hat{\xi}_{j}$, where $\delta=\|\boldsymbol{\xi}\|-\|\hat{\boldsymbol{\xi}}\|>0$ (the inequality follows from our initial assumption), resulting

$$
\dot{v}_{j}\left(\hat{\xi}_{j}\right)<p(\|\boldsymbol{\xi}\|)+\hat{\xi}_{j} \dot{p}(\|\boldsymbol{\xi}\|)+\delta \dot{p}(\|\boldsymbol{\xi}\|)
$$

Now, we can use our hypothesis $\xi_{j}>\hat{\xi}_{j}$ and (24) to obtain an upper bound of (25)

$$
p(\|\boldsymbol{\xi}\|)+\hat{\xi}_{j} \dot{p}(\|\boldsymbol{\xi}\|)<p(\|\boldsymbol{\xi}\|)+\xi_{j} \dot{p}(\|\boldsymbol{\xi}\|)=\dot{v}_{j}\left(\xi_{j}\right) .
$$

Thus, from (25) and (26) we have

$$
\dot{v}_{j}\left(\hat{\xi}_{j}\right)<\dot{v}_{j}\left(\xi_{j}\right)+\delta \dot{p}(\|\boldsymbol{\xi}\|) .
$$

From Assumption 1 we know that $\dot{p}(\|\boldsymbol{\xi}\|)>0$. Since $\delta>0$, 25 implies that $\hat{\xi}_{j}>\xi_{j}$, contradicting our initial assumption. Therefore we conclude that the total demand cannot decrease with the number of users, i.e., $\|\boldsymbol{\xi}\| \leq\|\hat{\boldsymbol{\xi}}\|$.

The following result shows some conditions to guarantee that all users have a positive demand.

Lemma 2. Suppose that Assumption 1 is satisfied. If all users have the same valuation function $\left(v_{i}(x)=v(x)\right.$ for all $\left.x \geq 0\right)$ and $\dot{v}(0)>p(0)$, then they will have positive demand in the equilibrium, i.e., $\xi_{i}>0$ for all $i \in \mathcal{P}$.

Proof. If all users have the same valuation function, then they will have the same demand, that is, $\xi_{i}=$ $\xi$ for all $i \in \mathcal{P}$ and $\|\boldsymbol{\xi}\|=N \xi$. Therefore, the equilibrium conditions in (9) become

$$
\begin{cases}\xi=0 & \text { if } \dot{v}(0)<p(0) \\ \dot{v}(\xi)=p(N \xi)+\xi \dot{p}(N \xi) & \text { Otherwise. }\end{cases}
$$

Observe that $\dot{v}(\xi)$ is decreasing and $p(N \xi)+\xi \dot{p}(N \xi)$ is increasing (see Assumption 21). Therefore, if $\dot{v}(0)>p(0)$, then there exists some $\xi>0$ such that $\dot{v}(\xi)=p(N \xi)+\xi \dot{p}(N \xi)$. 
In order to analyze the case in which every user has a positive demand, we assume that all users have the same valuation with $\dot{v}_{i}(0)>p(0)$ for all in $i \in \mathcal{P}$. In this way we guarantee that $\xi_{i}>0$ (see Lemma 2). The next Corollary shows that under the previous conditions the total demand strictly increases with the size of the population $N$.

Corollary 1. Consider the conditions of Proposition 4. If all users have identical valuation functions with $\dot{v}_{i}(0)>p(0)$, then the total demand in the NE strictly increases with the number of users, that is, $\|\boldsymbol{\xi}\|<\|\hat{\boldsymbol{\xi}}\|$.

Proof. Let $\mathcal{P}=\{1, \ldots, N\}$ and $\hat{\mathcal{P}}=\mathcal{P} \cup\{N+1\}$. Let all the users have the same valuation function, that is, $v_{i}(x)=v_{j}(x)=v(x)$ for all $x \in \mathbb{R}$ and $i, j \in \hat{\mathcal{P}}$. Consequently, all users will have the same demand, in other words, $\xi_{i}=\xi i \in \mathcal{P}$ and $\hat{\xi}_{j}=\hat{\xi}$ for all $j \in \hat{\mathcal{P}}$ and the total demand for each system will be equal to $\|\boldsymbol{\xi}\|=N \xi$ and $\|\hat{\xi}\|=(N+1) \hat{\xi}$. Furthermore, if $\dot{v}(0)>p(0)$, then $\xi, \hat{\xi} \neq 0$ (see Lemma 2).

Let us assume by contradiction that both systems have the same demand, i.e., $\|\boldsymbol{\xi}\|=\|\hat{\boldsymbol{\xi}}\|=g$, which leads to $\frac{N}{N+1} \xi=\hat{\xi}$. Therefore, the larger population consumes les energy, that is, $\xi>\hat{\xi}$. Hence, from the equilibrium conditions in (9) we get

$$
\dot{v}(\hat{\xi})=p(g)+\hat{\xi} \dot{p}(g)<p(g)+\xi \dot{p}(g)=\dot{v}(\xi) .
$$

The previous expression implies that $\hat{\xi}$ is greater than $\xi$, which contradicts our initial hypothesis. Therefore, $\|\boldsymbol{\xi}\| \neq\|\hat{\boldsymbol{\xi}}\|$, and from Proposition 4 we conclude that when all users have the same valuation the total demand strictly increases with the number of users, that is, $\|\boldsymbol{\xi}\|<\|\hat{\boldsymbol{\xi}}\|$.

Now, we show that if the marginal cost function $\dot{C}(\cdot)$ is unbounded then the total demand reaches a boundary as the population grows. If the cost function is bounded, then users would consume any amount of energy without paying significant prices.

Lemma 3. Suppose that Assumption 1 is satisfied. If $\dot{C}(\cdot)$ is unbounded, then the total demand reaches an upper bound as the number of users increase. That is, $\|\boldsymbol{\mu}\| \rightarrow K_{o}$ and $\|\boldsymbol{\xi}\| \rightarrow K_{s}$ as $N \rightarrow \infty$, for $K_{o}, K_{s} \in \mathbb{R}$. Moreover, if all users have the same valuation function with $\dot{v}_{i}(0)>p(0)$, then the individual demand decreases as the population grows, that is, $\mu_{i} \rightarrow 0$ and $\xi_{i} \rightarrow 0$ as $N \rightarrow \infty$.

Proof. Let us assume by contradiction that the optimal aggregate demand $\|\boldsymbol{\mu}\|$ is unbounded, that is, $\|\boldsymbol{\mu}\| \rightarrow \infty$ as $N \rightarrow \infty$. Hence, for every user $i$ with positive demand $\mu_{i}>0$ the following equilibrium condition follows from (7)

$$
\lim _{N \rightarrow \infty} \dot{v}_{i}\left(\mu_{i}\right)=\lim _{N \rightarrow \infty} \dot{C}(\|\boldsymbol{\mu}\|) .
$$

If $\dot{C}(\cdot)$ is unbounded, then

$$
\lim _{g \rightarrow \infty} \dot{C}(g) \rightarrow \infty
$$

Hence, our initial hypothesis imply that $\dot{v}_{i}\left(\mu_{i}\right) \rightarrow$ $\infty$ as $N \rightarrow \infty$, which contradicts Assumption 1 because $v(\cdot)$ is not differentiable. Therefore, we conclude that if $\dot{C}(\cdot)$ is unbounded, then the total demand is bounded, that is, there exists some $K_{o} \in \mathbb{R}$ such that $\|\boldsymbol{\mu}\| \rightarrow K_{o}$ as $N \rightarrow \infty$. Moreover, we can use a similar argument to prove that the aggregate demand in the NE $(\|\boldsymbol{\xi}\|)$ is also upper bounded by some positive number $K_{s}$.

Besides, if all users have the same valuation function, i.e., $v_{i}(x)=v_{j}(x)=v(x)$ for all $x \in \mathbb{R}$ and $i, j \in \mathcal{P}$, then they have the same demand, that is, $\xi_{i}=\bar{\xi}$ and $\mu_{i}=\bar{\mu}$ for all $i \in \mathcal{P}$. If the total demand is bounded, then

$$
\lim _{N \rightarrow \infty} \bar{\mu}=\lim _{N \rightarrow \infty} \frac{\|\boldsymbol{\mu}\|}{N} \leq \lim _{N \rightarrow \infty} \frac{K_{o}}{N}=0 .
$$

Therefore, the demand of each user $\bar{\mu}$ tends to zero as $N$ grows. Likewise, $\bar{\xi} \rightarrow 0$ as $N \rightarrow \infty$.

With the previous results we can prove that the demand ratio $\|\boldsymbol{\xi}\| /\|\boldsymbol{\mu}\|$ reaches an upper bound as the number of users increases (if the price function $p(\cdot)$ is linear $)$.

Proof. If $v_{i}(x)=v_{j}(x)=v(x)$ for all $x \in \mathbb{R}$ and $i, j \in \mathcal{P}$, then all users have the same demand, that is, $\xi_{i}=\bar{\xi}$ and $\mu_{i}=\bar{\mu}$ for all user $i \in \mathcal{P}$. From Lemma 3 the demand of users tends to zero as the number of users increase. Hence, the equilibrium conditions in (7) and (9) lead to

$$
\lim _{N \rightarrow \infty} \dot{v}_{i}(\bar{\xi})=\lim _{\bar{\xi} \rightarrow 0} \dot{v}(\bar{\xi})=p(\|\boldsymbol{\xi}\|)
$$

and

$$
\lim _{N \rightarrow \infty} \dot{v}_{i}(\bar{\mu})=\lim _{\bar{\mu} \rightarrow 0} \dot{v}(\bar{\mu})=\dot{C}(\|\boldsymbol{\mu}\|) .
$$

From (27) and (28) we obtain

$$
\dot{v}(0)=p(\|\boldsymbol{\xi}\|)=p(\|\boldsymbol{\mu}\|)+\|\boldsymbol{\mu}\| \dot{p}(\|\boldsymbol{\mu}\|) .
$$

If $p(\cdot)$ is linear then $\|\boldsymbol{\xi}\|=2\|\boldsymbol{\mu}\|$. 
The next theorem shows that the price of anarchy equals zero, that is, the customer surplus in the worst case NE is arbitrarily smaller than the social optimum.

Proof of Theorem [3. Observe that only users with positive demand affect the efficiency ratio, because $U_{i}\left(0, \boldsymbol{q}_{-i}\right)=0($ see (5) $)$. Therefore, henceforth we assume that $\mu_{i}>0$.

Now, let us find an upper bound of the aggregate valuations at the Nash and the optimal equilibria. On one hand, from the concavity of the valuation functions we have $v_{i}\left(\mu_{i}\right) \geq v_{i}(0)+\mu_{i} \dot{v}_{i}\left(\mu_{i}\right)$. Since $\dot{v}_{i}(0)=0$, then $v_{i}\left(\mu_{i}\right) \geq \mu_{i} \dot{v}_{i}\left(\mu_{i}\right)$. Summing over all users and using the conditions of the optimal equilibrium in (7) we obtain

$$
\sum_{i} v_{i}\left(\mu_{i}\right) \geq\|\boldsymbol{\mu}\| p(\|\boldsymbol{\mu}\|)+\|\boldsymbol{\mu}\|^{2} \dot{p}(\|\boldsymbol{\mu}\|) .
$$

On the other hand, from the concavity of the valuation functions we obtain $v_{i}\left(\xi_{i}\right) \geq v_{i}\left(\mu_{i}\right)+\left(\xi_{i}-\right.$ $\left.\mu_{i}\right) \dot{v}_{i}\left(\xi_{i}\right)$. Likewise, summing over all agents and using the conditions of the Nash equilibrium in (9) results 16

$$
\sum_{i} v_{i}\left(\xi_{i}\right) \geq \sum_{i} v_{i}\left(\mu_{i}\right)+A(\boldsymbol{\xi}, \boldsymbol{\mu})+B(\boldsymbol{\xi}, \boldsymbol{\mu}),
$$

where $A(\boldsymbol{\xi}, \boldsymbol{\mu})=(\|\boldsymbol{\xi}\|-\|\boldsymbol{\mu}\|) p(\|\boldsymbol{\xi}\|)$ and $B(\boldsymbol{\xi}, \boldsymbol{\mu})=$ $\sum_{i} \xi_{i}\left(\xi_{i}-\mu_{i}\right) \dot{p}(\|\boldsymbol{\xi}\|)$. Since $\xi_{i} \geq 0$ and $\|\boldsymbol{\xi}\| \geq\|\boldsymbol{\mu}\|$, we can obtain the following lower bound of $B(\boldsymbol{\xi}, \boldsymbol{\mu})$

$$
B(\boldsymbol{\xi}, \boldsymbol{\mu}) \geq \underline{\xi}(\|\boldsymbol{\xi}\|-\|\boldsymbol{\mu}\|)=\hat{B}(\boldsymbol{\xi}, \boldsymbol{\mu})
$$

With (30) and (31) we express the efficiency ratio as

$$
\begin{aligned}
r(\boldsymbol{\xi}, \boldsymbol{\mu})= & \frac{\sum_{i} v_{i}\left(\xi_{i}\right)-\|\boldsymbol{\xi}\| p(\|\boldsymbol{\xi}\|)}{\sum_{i} v_{i}\left(\mu_{i}\right)-\|\boldsymbol{\mu}\| p(\|\boldsymbol{\mu}\|)} \\
& \geq \frac{\sum_{i} v_{i}\left(\mu_{i}\right)+\Gamma(\boldsymbol{\xi}, \boldsymbol{\mu})-C(\|\boldsymbol{\xi}\|)}{\sum_{i} v_{i}\left(\mu_{i}\right)-C(\|\boldsymbol{\mu}\|)},
\end{aligned}
$$

where $\Gamma(\boldsymbol{\xi}, \boldsymbol{\mu})=A(\boldsymbol{\xi}, \boldsymbol{\mu})+\hat{B}(\boldsymbol{\xi}, \boldsymbol{\mu})$.

Let us represent the right hand side of (32) as the following function that depends on the total valuation $\sum_{i} v_{i}\left(\mu_{i}\right)$

$$
\Xi(x)=\frac{x-y}{x-z},
$$

\footnotetext{
${ }^{16}$ Observe that 29 and 30 hold with equality when the valuation functions are linear.
}

where $x=\sum_{i} v_{i}\left(\mu_{i}\right), y=C(\|\boldsymbol{\xi}\|)-\Gamma(\boldsymbol{\xi}, \boldsymbol{\mu})$ and $z=C(\|\boldsymbol{\mu}\|)$. We know that $1 \geq \Xi(x)$, which implies that $y \geq z$; therefore, the function $\Xi(x)$ is increasing with respect to $x$.

The function in (33) help us to find a lower bound to the efficiency ratio. Observe that $\Xi(x) \geq \Xi(x-\epsilon)$, for $\epsilon \geq 0$. Hence, we obtain a lower bound replacing $\sum_{i} v_{i}\left(\mu_{i}\right)$ by its lower bound in (29), resulting

$$
\Xi(x) \geq \frac{\|\boldsymbol{\mu}\|^{2} \dot{p}(\|\boldsymbol{\mu}\|)+\hat{B}(\boldsymbol{\xi}, \boldsymbol{\mu})-D(\boldsymbol{\xi}, \boldsymbol{\mu})}{\|\boldsymbol{\mu}\|^{2} \dot{p}(\|\boldsymbol{\mu}\|)},
$$

where $D(\boldsymbol{\xi}, \boldsymbol{\mu})=\|\boldsymbol{\mu}\|(p(\|\boldsymbol{\xi}\|)-p(\|\boldsymbol{\mu}\|))$. Note that (34) holds with equality when the valuation functions are linear, because in that case the inequality in (29) holds with equality.

Finally, if $p(\cdot)$ is linear, then the right hand side of (34) becomes

$$
\begin{aligned}
& \Xi(x) \geq \tilde{r}(\boldsymbol{\xi}, \boldsymbol{\mu}) \\
& \quad=\left(\|\boldsymbol{\mu}\|^{2}+(\|\boldsymbol{\xi}\|-\|\boldsymbol{\mu}\|)(\underline{\xi}-\|\boldsymbol{\mu}\|)\right) \frac{1}{\|\boldsymbol{\mu}\|} .
\end{aligned}
$$

From Lemma 3 we know that if $\dot{C}(\cdot)$ is unbounded and all users have the same valuation, then $\xi_{i} \rightarrow 0$ as $N \rightarrow \infty$. Therefore, $\xi \rightarrow 0$, which leads (35) to

$$
\tilde{r}(\boldsymbol{\xi}, \boldsymbol{\mu}) \rightarrow 2-\eta,
$$

where $\eta=\|\boldsymbol{\xi}\| /\|\boldsymbol{\mu}\|$. From Lemma 1 we know that when all users have the same valuation $\eta \rightarrow 2$ as $N \rightarrow \infty$, which makes (36) equal to zero. Hence, the worst efficiency loss occurs when all users have the same linear valuation function, the price $p(\cdot)$ is linear, and $N \rightarrow \infty$.

\subsection{Properties of the Mechanism}

This subsection shows results related with the impossibility of having a mechanism that satisfies budget balance and we prove the properties of two instances of the mechanism.

Let us introduce some notation for the following results. Let $\theta=\sum_{i \in \mathcal{P}}\left\|\boldsymbol{q}_{-i}\right\|=(N-1)\|\boldsymbol{q}\|$ and $\rho_{i}=\left\|\boldsymbol{q}_{-i}\right\| / \theta$, which satisfies $\sum_{i \in \mathcal{P}} \rho_{i}=1$. The previous variables allow us to rewrite the total incentives as

$\sum_{i \in \mathcal{P}} I_{i}(\boldsymbol{q})=\theta\left(\sum_{i \in \mathcal{P}} \rho_{i} h\left(\rho_{i} \theta\right)-p\left(\frac{\theta}{N-1}\right)\right)$.

The following proof shows that it is not possible to have mechanisms with the budget balance property. 
Proof of Theorem 4. Let us assume by contradiction that there exists some function $h(\cdot)$ such that the incentives satisfy $\sum_{i \in \mathcal{P}} I_{i}(\boldsymbol{q})=0$. Therefore, we can rewrite (37) as

$$
p\left(\frac{\theta}{N-1}\right)=\sum_{i \in \mathcal{P}} \rho_{i} h\left(\rho_{i} \theta\right) .
$$

Now, we consider two scenarios. First, if all users have the same consumption, represented by the vector $\hat{\boldsymbol{\rho}}$ with $\hat{\rho}_{i}=\hat{\rho}_{j}=\frac{1}{N}$ for all $i, j \in \mathcal{P}$, then, (38) becomes

$$
p\left(\frac{\theta}{N-1}\right)=h\left(\frac{\theta}{N}\right) .
$$

Second, if all except one user have positive consumption, represented by the vector $\tilde{\boldsymbol{\rho}}$ with $\tilde{\rho}_{i}=0$ and $\tilde{\rho}_{j}=\frac{1}{N-1}$ for all $j \neq i$, then, assuming that $\lim _{\rho \rightarrow 0} \rho_{j} h\left(\rho_{j} \theta\right)=0$ (38) becomes

$$
p\left(\frac{\theta}{N-1}\right)=h\left(\frac{\theta}{N-1}\right) .
$$

Equations (39) and (40) are valid only when $\theta=0$ (for $N$ finite). Therefore, we find a contradiction and conclude that we cannot find a function $h(\cdot)$ that satisfies the budget balance property.

The next result shows the conditions to have incentive compatibility in a mechanism.

Proof of Proposition 1. Let us consider a vector $\hat{\boldsymbol{\mu}}$ equal to $\boldsymbol{\mu}$ except for its $i^{\text {th }}$ entry, which is equal to zero. Hence, $\hat{\mu}_{j}=\mu_{j}$ for all $j \neq i$ and $\hat{\mu}_{i}=0$, which leads to $\|\hat{\boldsymbol{\mu}}\|=\left\|\hat{\boldsymbol{\mu}}_{-i}\right\|=\left\|\boldsymbol{\mu}_{-i}\right\|$. From the optimality condition in (7) we know that

$$
\begin{aligned}
& W_{i}(\boldsymbol{\mu}) \geq W_{i}(\hat{\boldsymbol{\mu}}) \\
& \quad=I_{i}(\hat{\boldsymbol{\mu}})=\left\|\boldsymbol{\mu}_{-i}\right\|\left(h\left(\left\|\boldsymbol{\mu}_{-i}\right\|\right)-p\left(\left\|\boldsymbol{\mu}_{-i}\right\|\right)\right) .
\end{aligned}
$$

Therefore, if $h\left(\left\|\boldsymbol{\mu}_{-i}\right\|\right)-p\left(\left\|\boldsymbol{\mu}_{-i}\right\|\right) \geq 0$, then $W_{i}(\boldsymbol{\mu}) \geq 0$.

The next result shows an instance of the proposed mechanism that has budget deficit.

Proof of Proposition 2. Observe that $h(\cdot)$ in (13) is increasing, because $p(\cdot)$ is increasing. Therefore,

$$
I_{i}(\boldsymbol{q})=\left\|\boldsymbol{q}_{-i}\right\|\left(p\left(\left\|\boldsymbol{q}_{-i}\right\|\right)-p(\|\boldsymbol{q}\|)\right) \leq 0 .
$$

Hence, we conclude that $\sum_{i \in \mathcal{P}} I_{i}(\boldsymbol{q}) \leq 0$.

Moreover, $h\left(\left\|\boldsymbol{\mu}_{-i}\right\|\right)=p\left(\left\|\boldsymbol{\mu}_{-i}\right\|\right)$, satisfying the conditions of Proposition 1 .
The next result shows an instance of the proposed mechanism that has budget surplus.

Proof of Proposition 3. With (14) we can write

$$
\rho_{i} h\left(\rho_{i} \theta\right)=\frac{z_{i}}{k \theta} p\left(z_{i}\right),
$$

where $z_{i}=\theta k \rho_{i}$ and $k=\frac{N}{N-1}$. Let $t(g)=g p(g)$, which is convex (see Assumption 2), then

$$
\sum_{i \in \mathcal{P}} \frac{z_{i}}{k \theta} p\left(z_{i}\right)=\sum_{i \in \mathcal{P}} \frac{N}{N k \theta} t\left(z_{i}\right) \geq \frac{N}{k \theta} t\left(\frac{1}{N} \sum_{i \in \mathcal{P}} z_{i}\right) .
$$

Here we have that $\sum_{i \in \mathcal{P}} z_{i}=k \theta$, therefore (41) becomes

$$
\sum_{i \in \mathcal{P}} \frac{z_{i}}{k \theta} p\left(z_{i}\right) \geq p\left(\frac{\theta}{N-1}\right) .
$$

We can use the lower bound (42) in (37) to show that $\sum_{i \in \mathcal{P}} I_{i}(\boldsymbol{q}) \geq 0$.

Moreover, we know from Proposition 1 that this mechanism is individually rational since $h\left(\left\|\boldsymbol{\mu}_{-i}\right\|\right)=p\left(\frac{N}{N-1}\left\|\boldsymbol{\mu}_{-i}\right\|\right) \geq p\left(\left\|\boldsymbol{\mu}_{-i}\right\|\right)$. The inequality follows since $p(\cdot)$ is increasing (see Assumption 11).

\subsection{Experimental Setup}

The stability of the system depends on its dynamics, i.e., on how customers update their actions in response to price changes. We assume that each customer uses a load management system that finds the action (i.e., demand) that maximizes either $U_{i}$ or $W_{i}$ (if the customers receive incentives). Here we leverage the theory of population games to define the system's dynamics that guarantee stability and convergence to either $\boldsymbol{\xi}$ or $\boldsymbol{\mu}$ (in the case with incentives).

In particular, we assume that each load management system has built-in some evolutionary $d y$ namics configured maximize the customer's profit 51, 52, 53. The evolutionary dynamics describe how populations of agents, who participate in a game, update their strategies to maximize a fitness function. We can maximize the customer's profit making the fitness function equal to the marginal profit; thus, the evolutionary dynamics resemble a distributed gradient based optimization method [54, 55]. We use the evolutionary dynamics because, thanks to the concavity of the surplus functions, we 
can guarantee that the system is asymptotically stable.

We configure the evolutionary dynamics as follows. Each customer has some resources (e.g., energy) and decides whether to use them to perform tasks (e.g., turn on appliances). In other words, the customers have two strategies, whether to use or not the resources. In this case, the benefit of using the resources equals to the marginal profit. On the contrary, idle resources do not report benefits nor losses. Thus, in the equilibrium both strategies give the same benefit, that is, the dynamics balance the benefit between both strategies. This also implies that in the equilibrium the marginal profit equals to zero, as required to satisfy the FOC.

We find the optimal equilibrium and the Nash equilibrium using the Population Dynamics Toolbox in [56]. We refer the interested reader to [57] for more details on the implementation.

\section{References}

[1] J. S. Vardakas, N. Zorba, C. V. Verikoukis, A survey on demand response programs in smart grids: Pricing methods and optimization algorithms, IEEE Communications Surveys Tutorials 17 (1) (2015) 152-178. doi:10.1109/COMST.2014.2341586

[2] D. E. Aliabadi, M. Kaya, G. Sahin, Competition, risk and learning in electricity markets: An agent-based simulation study, Applied energy 195 (2017) 1000-1011.

[3] D. Paredes, S. Loveridge, Rural electric cooperatives and economic development, Energy Policy 117 (2018) $49-57$.

[4] I. Heras-Saizarbitoria, L. Sáez, E. Allur, J. Morandeira, The emergence of renewable energy cooperatives in spain: A review, Renewable and Sustainable Energy Reviews 94 (2018) 1036-1043.

[5] I. Capellán-Pérez, Á. Campos-Celador, J. TerésZubiaga, Renewable energy cooperatives as an instrument towards the energy transition in spain, Energy Policy 123 (2018) 215-229.

[6] B. Huybrechts, S. Mertens, The relevance of the cooperative model in the field of renewable energy, Annals of Public and Cooperative Economics 85 (2) (2014) 193212.

[7] European federation rescoop.eu accessed January 3, 2019 (2018) URL https://www.rescoop.eu/federation

[8] R. Deng, Z. Yang, M.-Y. Chow, J. Chen, A survey on demand response in smart grids: Mathematical models and approaches, IEEE Transactions on Industrial Informatics 11 (3) (2015) 570-582.

[9] J. Lazar, F. Weston, W. Shirley, J. MigdenOstrander, D. Lamont, E. Watson, Revenue regulation and decoupling: A guide to theory Tech. rep., The Regulatory Assistance Project (RAP) (2016).

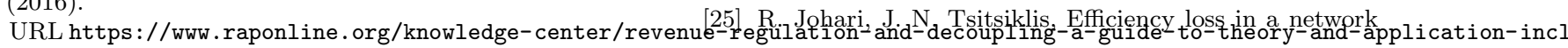

[10] A. Mohsenian-Rad, V. Wong, J. Jatskevich, R. Schober, A. Leon-Garcia, Autonomous Demand-Side Management Based on Game-Theoretic Energy Consumption Scheduling for the Future Smart Grid, IEEE Transactions on Smart Grid 1 (3) (2010) 320-331. doi:10.1109/TSG.2010.2089069

[11] L. Chen, N. Li, S. H. Low, J. C. Doyle, Two market models for demand response in power networks, in: 2010 First IEEE International Conference on Smart Grid Communications (SmartGridComm), IEEE, 2010, pp. 397-402.

[12] S. Li, W. Zhang, J. Lian, K. Kalsi, Market-based coordination of thermostatically controlled loads - part i: A mechanism design formulation, IEEE Transactions on Power Systems 31 (2) (2016) 1170-1178.

[13] H. Chen, Y. Li, R. H. Louie, B. Vucetic, Autonomous demand side management based on energy consumption scheduling and instantaneous load billing: An aggregative game approach, IEEE Transactions on Smart Grid 5 (4) (2014) 1744-1754.

[14] L. C. Corchon, Comparative statics for aggregative games the strong concavity case, Mathematical Social Sciences 28 (3) (1994) 151-165.

[15] A. De Paola, D. Angeli, G. Strbac, Convergence and optimality of a new iterative price-based scheme for distributed coordination of flexible loads in the electricity market, in: Decision and Control (CDC), 2017 IEEE 56th Annual Conference on, IEEE, 2017, pp. 1386-1393.

[16] E. Koutsoupias, C. Papadimitriou, Worst-case equilibria, in: Annual Symposium on Theoretical Aspects of Computer Science, Springer, 1999, pp. 404-413.

[17] D. P. Zhou, M. Roozbehani, M. A. Dahleh, C. J. Tomlin, Stability analysis of wholesale electricity markets under dynamic consumption models and real-time pricing, in: American Control Conference (ACC), 2017, IEEE, 2017, pp. 2048-2053.

[18] M. Roozbehani, M. A. Dahleh, S. K. Mitter, Volatility of power grids under real-time pricing, IEEE Transactions on Power Systems 27 (4) (2012) 1926-1940.

[19] C. Barreto, A. Cardenas, Impact of the market infrastructure on the security of smart grids, IEEE Transactions on Industrial Informatics (2018) 1doi:10.1109/TII.2018.2886292

[20] S. Soltan, P. Mittal, H. V. Poor, Blackiot: Iot botnet of high wattage devices can disrupt the power grid, in: 27th USENIX Security Symposium (USENIX Security 18), USENIX Association, Baltimore, MD, 2018, pp. 1532 .

[21] M. Negrete-Pincetic, F. Yoshida, G. Gross, Towards quantifying the impacts of cyber attacks in the competitive electricity market environment, in: 2009 IEEE PowerTech, 2009, pp. 1-8.

[22] Y. Liu, S. Hu, T.-Y. Ho, Vulnerability assessment and defense technology for smart home cybersecurity considering pricing cyberattacks, in: 2014 IEEE/ACM International Conference on Computer-Aided Design (ICCAD), IEEE, 2014, pp. 183-190.

[23] J. Liyan, R. J. Thomas, L. Tong, Impacts of malicious data on real-time price of electricity market operations, in: 45th Hawaii International Conference on System Sciences, 2012, pp. pp.1907-1914.

1. Toughgarden, E. Tardos, How Bad is Selfish Routing?, Journal of the ACM (JACM) 49 (2) (2002) 236259. doi:10.1145/506147.506153 
resource allocation game, Mathematics of Operations Research 29 (3) (2004) 407-435.

[26] R. Johari, J. N. Tsitsiklis, A scalable network resource allocation mechanism with bounded efficiency loss, IEEE Journal on Selected Areas in Communications 24 (5) (2006) 992-999.

[27] P. Samadi, H. Mohsenian-Rad, R. Schober, V. W. S. Wong, Advanced demand side management for the future smart grid using mechanism design, IEEE Transactions on Smart Grid 3 (3) (2012) 1170-1180. doi:10.1109/TSG.2012.2203341

[28] W. H. Sandholm, Negative externalities and evolutionary implementation, The Review of Economic Studies 72 (3) (2005) 885-915.

[29] A. Kakhbod, D. Teneketzis, An efficient game form for unicast service provisioning, IEEE Transactions on Automatic Control 57 (2) (2012) 392-404.

[30] A. Kakhbod, D. Teneketzis, An efficient game form for multi-rate multicast service provisioning, IEEE Journal on Selected Areas in Communications 30 (11) (2012) 2093-2104.

[31] S. Reichelstein, S. Reiter, Game forms with minimal message spaces, Econometrica: Journal of the Econometric Society (1988) 661-692.

[32] P. J. Healy, L. Mathevet, Designing stable mechanisms for economic environments, Theoretical economics 7 (3) (2012) 609-661.

[33] N. Nisan, T. Roughgarden, E. Tardos, V. V. Vazirani, Algorithmic Game Theory, Cambridge University Press, 32 Avenue of the Americas, New York, NY 10013-2473, USA, 2007.

[34] T. Groves, J. O. Ledyard, Optimal Allocation of Public Goods: A Solution to the "Free Rider" Problem, Econometrica 45 (4) (1977) 783-809.

[35] C. Barreto, E. Mojica-Nava, N. Quijano, Design of mechanisms for demand response programs, in: 52nd IEEE Conference on Decision and Control, IEEE, 2013, pp. 1828-1833. doi:10.1109/CDC.2013.6760148

[36] C. Barreto, A. A. Cárdenas, Incentives for demandresponse programs with nonlinear, piece-wise continuous electricity cost functions, in: 2015 American Control Conference (ACC), IEEE, 2015, pp. 4327-4332. doi:10.1109/ACC.2015.7172009

[37] S. Boyd, L. Vandenberghe, Convex optimization, Cambridge university press, 2004.

[38] H. Kuhn, A. Tucker, Nonlinear programming, in: Proceedings of 2nd Berkeley Symposium, University of California Press, 1951, pp. 481-492.

[39] W. Karush, Minima of functions of several variables with inequalities as side constraints, M. Sc. Dissertation. Dept. of Mathematics, Univ. of Chicago.

[40] A. Mas-Colell, M. D. Whinston, J. R. Green, Microeconomic theory, Vol. 1, Oxford university press New York,
1995.

[41] D. S. Kirschen, G. Strbac, Fundamentals of power system economics, John Wiley \& Sons, 2004.

[42] A.-A. Cournot, Recherches sur les principes mathématiques de la théorie des richesses par $\mathrm{Au}-$ gustin Cournot, chez L. Hachette, 1838.

[43] J. Nash, Non-cooperative games, Annals of mathematics (1951) 286-295.

[44] D. Fudenberg, J. Tirole, Game Theory, MIT Press, 1991.

[45] J. B. Rosen, Existence and uniqueness of equilibrium points for concave n-person games, Econometrica: Journal of the Econometric Society (1965) 520-534

[46] G. Hardin, The tragedy of the commons, Science 162 (3859) (1968) 1243-1248.

[47] C. Papadimitriou, Algorithms, games, and the internet, in: Proceedings of the thirty-third annual ACM symposium on Theory of computing, ACM, 2001, pp. 749-753.

[48] L. Hurwicz, S. Reiter, Designing Economic Mechanisms, Cambridge University Press, 2006.

[49] D. Monderer, L. S. Shapley, Potential games, Games and economic behavior 14 (1) (1996) 124-143.

[50] R. B. Myerson, M. A. Satterthwaite, Efficient mechanisms for bilateral trading, Journal of Economic Theory 29 (2) (1983) 265-281.

[51] W. H. Sandholm, Population Games and Evolutionary Dynamics (Economic Learning and Social Evolution), 1st Edition, The MIT Press, 2011.

[52] J. Hofbauer, From nash and brown to maynard smith equilibria, dynamics and ess, Selection 1 (1) (2000) 8188.

[53] N. Quijano, C. Ocampo-Martinez, J. Barreiro-Gomez, G. Obando, A. Pantoja, E. Mojica-Nava, The role of population games and evolutionary dynamics in distributed control systems: The advantages of evolutionary game theory, IEEE Control Systems Magazine 37 (1) (2017) 70-97. doi:10.1109/MCS.2016.2621479

[54] A. Pantoja, N. Quijano, A Population Dynamics Approach for the Dispatch of Distributed Generators, IEEE Transactions on Industrial Electronics 58 (10) (2011) 4559-4567. doi:10.1109/TIE.2011.2107714.

[55] E. Mojica-Nava, C. Barreto, N. Quijano, Population games methods for distributed control of microgrids, IEEE Transactions on Smart Grid 6 (6) (2015) 25862595. doi:10.1109/TSG.2015.2444399

[56] C. Barreto, Population dynamics toolbox (pdtoolbox), https://github.com/carlobar/PDToolbox_matlab accessed: April 10, 2018 (2014).

[57] C. Barreto, E. Mojica-Nava, N. Quijano, Incentives-based mechanism for efficient demand response programs arXiv preprint arXiv:1408.5366Submitted. URL https://arxiv.org/abs/1408.5366 